\title{
Effects of visual field of presentation and stimulus characteristics on visual discrimination learning*
}

\author{
PATRICIA Y. LeFEBVRE and SUNNAN K. KUBOSE \\ University of North Carolina at Greensboro, Greensboro, North Carolina 27412
}

\begin{abstract}
If reversal behavior requires reference to verbal abilities, then response latencies should be shorter when the discrimination stimuli have direct access to the brain areas concerned with these functions. Stimuli were tachistoscopically presented to the left, center, and right visual fields. Results indicated that during the initial discrimination, verbal tasks were not affected by field of presentation, but nonverbal tasks had significantly shorter reactions times with left field presentation. For the reversal discrimination, right field presentation resulted in significantly shorter response latencies for both tasks.
\end{abstract}

To describe adult discrimination learning, Kendler and Kendler (1962) suggest a mediational model consisting of two integrated S-R units in which the external stimulus is followed by an implicit response that evokes an implicit cue connected to an overt response. Kendler (1964) further suggests that the existence of this covert linkage is determined by a verbal factor. In this sense, a verbal label may act as the symbolic or implicit cue preceding the overt response. Studies (Kendler, Kendler, \& Wells, 1960; Kendler, 1964; Kendler, Kendler, \& Saunders, 1967) present evidence implicating the role of language in the execution of mediated responses. This evidence is, however, inconclusive in identifying the specific role of language during mediated behavior.

The present research investigated the possible relationship between field of visual presentation and discrimination behavior. If mediation is facilitated by accessibility to verbal processes, then information must travel to the areas of the brain concerned with this function. There is ample evidence for-localization of the speech function within the dominant hemisphere (Penfield \& Roberts, 1959; McAdams \& Whitaker, 1971; Filbey \& Gazzaniga, 1969). Following this rationale, the present research utilized a modified reversal shift paradigm in which verbal and nonverbal stimuli were presented in either the left, center, or right visual field. Stimuli which require reference to verbal areas entering the minor hemisphere (left visual field) must be transmitted to the speech areas located on the dominant side. If execution of reversal shifts (mediational behavior) requires reference to the speech areas of the brain, then left visual field presentation should result in longer reaction times.

\section{METHOD}

\footnotetext{
Subjects

Ss were 46 introductory psychology students from the University of North Carolina at Greensboro and eight adults not

*Sponsored by Robert G. Eason, who takes full editorial responsibility for the manuscript.
}

enrolled at the University. All Ss were of comparable ages. All Ss were right-handed.

\section{Apparatus}

The stimulus cards were presented in a two-channel Polymetric tachistoscope with a viewing distance of 17 in. Presentation of stimuli was coincident with the starting of a Standard Electric clock, and the S's response stopped the clock. Responses were made by flipping a switch with the right hand in the direction which corresponded spatially to the choice of stimuli.

\section{Design and Procedure}

The task was a two-choice, simultaneous visual discrimination requiring a reversal shift similar to the paradigm described by Kendler and Kendler (1962). The S first learned an initial discrimination which differed simultaneously on two dimensions; following this initial task, the $\mathrm{S}$ was presented with identical stimuli requiring a response along the same dimension but to a different cue on that dimension. The stimuli and task are illustrated in Table 1. For half the Ss, name was the relevant dimension, and for the other half, the relevant dimension was size. Half the group with the size task had "small" as positive; half had "large" as positive. For the name group, letter "a" or "A" was correct for half, and for half the group, letter name " $b$ " or "B" was correct. This counterbalancing procedure resulted in four experimental groups.

Stimuli were randomly presented to the left, center, and right visual fields with the left and right presentation having a visual angle of $3-1 / 3$ deg from a center fixation point. Each stimulus was presented for $200 \mathrm{msec}$. Ss were instructed to fixate on the center fixation point. They were told that when the stimulus pair was flashed on the screen they should decide which of the two was correct and move the switch in the direction to correspond to their choice. They were asked to respond as

Table 1

Experimental Design for Forced Reversal Shift Based on Name or Size Dimension

\begin{tabular}{|c|c|c|c|c|}
\hline & \multicolumn{2}{|c|}{$\begin{array}{c}\text { Initial } \\
\text { Discrimination }\end{array}$} & \multicolumn{2}{|c|}{$\begin{array}{c}\text { Reversal } \\
\text { Discrimination }\end{array}$} \\
\hline Size Dimension & $\begin{array}{c}+ \\
\mathbf{A} \\
+ \\
\mathbf{B}\end{array}$ & $\frac{\bar{b}}{\bar{a}}$ & $\begin{array}{l}\bar{A} \\
\bar{B}\end{array}$ & $\begin{array}{l}+ \\
b \\
+ \\
a\end{array}$ \\
\hline Name Dimension & $\frac{+}{\mathrm{A}}$ & $\begin{array}{l}\bar{b} \\
+ \\
\mathrm{a}\end{array}$ & $\begin{array}{l}\overline{\mathrm{A}} \\
+ \\
\mathrm{B}\end{array}$ & $\frac{t}{\mathrm{~b}}$ \\
\hline
\end{tabular}


quickly and as accurately as possible. Following the S's response, $E$ indicated whether the choice was correct or incorrect. Elapsed time for each response was recorded. Ss received trials on the initial discrimination until a criterion of 4 out of 5 correct was reached. Following criterion, an additional 12 trials with the same discrimination task were given. Then without warning, the reversal shift was initiated. Again, criterion was 4 out of 5 correct, with 12 trials following the attainment of criterion. Data from Ss not reaching criterion within 30 trials were not used.

During performance on the intital discrimination, no difference was expected as a function of field of presentation for the size task. This task should require no reference to verbal areas (Cohen, 1972). For the name task, a right visual-field superiority was expected in that such a task may require verbal processes. During performance on the reversal discrimination, a right-field superiority was expected for both the name and size tasks. If mediation does require reference to verbal processes, then direct access to the areas of the brain concerned with these processes should result in faster response latencies.

\section{RESULTS}

Analyses of variance were performed on the response latency data during acquisition and after criterion was attained for both the initial discrimination and for the reversal discrimination. Factors were field of presentation (a repeated measure), type of task (name or size), and counterbalancing. Additionally, an analysis of variance was performed for number of trials necessary to attain criterion for the initial and reversal discriminations.

The analysis of the acquisition latency data during the initial discrimination revealed no significant effects. For acquisition of the reversal discrimination, there was a significant effect due to field of presentation $(F=$ $11.0339 ; \mathrm{df}=2 / 88 ; \mathrm{p}<.01)$ and a significant interaction effect due to field of presentation and the counterbalancing procedure $(F=4.2052 ; \mathrm{df}=2 / 88$; $\mathrm{p}<.05)$. Tukey tests for the interaction showed that latencies for center-field presentation were significantly higher than left or right field of presentation when small or ' $\mathrm{Bb}$ ' was positive $(\mathrm{q}=.076801 ; \mathrm{r}=3 ; \mathrm{df}=88$; $\mathrm{p}<.01)$, while latencies for right and center presentation were significantly longer than left presentation when large or " $\mathrm{aA}$ " was positive $(\mathrm{q}=.05780$, $\overline{\mathrm{r}}=3 ; \mathrm{df}=88 ; \mathrm{p}<.05)$. Field of presentation during acquisition of the reversal discrimination accounted for $3 \%$ of the total variability, and the interaction accounted for $1 \%$ (based on utility index, Dodd \& Schultz, 1973).

The analysis of the postcriterion latency data for the initial discrimination indicated a significant main effect due to field of presentation $(F=13.196 ; \mathrm{df}=2 / 88$; $\mathrm{p}<.01)$ and a significant interaction effect for field of presentation and type of task $(F=7.65 ; \mathrm{df}=2 / 88$; $\mathrm{p}<.01)$. Tukey tests for differences between means showed that right-field presentation resuited in significantly longer response latencies than either left or center field $(\mathrm{q}=.03511 ; \mathrm{i}=\mathrm{j} ; \mathrm{df}=88 ; \mathrm{p}<.01)$. Tukey tests for the interaction indicated that for the name task, the effect of field of presentation was not significant; for the size task, the right field was inferior to both left and center fields $(q=.04966 ; r=3 ; d f=88 ; p<.01)$. Visual field of presentation accounted for $2 \%$ of the total variability, and the interaction of visual field and task accounted for $1 \%$ of the total variability. Calculation of the strength of association, after subtracting the variance due to Ss, showed that field of presentation accounted for $10 \%$ of the variability, and the interaction effect represented $5 \%$.

The analysis of variance for response latencies for postcriterion trails on the reversal discrimination showed a significant main effect due to field of presentation. Tukey tests indicated that left-field presentation resulted in significantly longer latencies than either center or right presentation $(\mathrm{q}=.02040 ; \mathrm{r}=3 ; \mathrm{df}=88 ; \mathrm{p}<.05)$. Field of presentation accounted for $7 \%$ of the total variance. Without the variance due to $\mathrm{Ss}$, field of presentation accounted for $50 \%$ of the variance.

Analysis of trials to criterion necessary for all conditions in both the initial and reversal discrimination showed that the name-size factor was significant $(F=$ $4.1800 ; \mathrm{df}=1 / 44 ; \mathrm{p}<.05)$. Ss having the size task generated a mean of 5.937 for trials necessary to attain criterion; whereas, the Ss having the name task required 7.605 trials to attain criterion. This factor accounted for $1 \%$ of the total variance.

\section{DISCUSSION}

For the postcriterion latencies in the size task during the initial discrimination phase, there is a clear left-visual field (right hemisphere) superiority, while in the reversal phase, this superiority is reversed, with significantly shorter response latencies for right-visual field presentation for both the name and size tasks. These different findings seem to indicate that there are different processes involved in the performance on the initial discrimination as compared to the reversal discrimination.

In relation to the hypothesis that performance of reversal discriminations involves a verbal process, results provide support. Congruent with lateralization of verbal functions within the left brain, right-field presentation of reversal stimuli does facilitate the discrimination process. During post-criterion trials on the reversal discrimination, there was a significant field effect. Both name and size discriminations were performed faster with right-field presentation. During the initial discrimination, however, there was a significant facilitiation of left-field presentation for the size task while the name task was performed equally well by both hemispheres. For the reversal shift, then, the major effect is due to the process of mediation rather than to the type of task.

\section{REFERENCES}

Cohen, G. Hemispheric differences in a letter classification task. Perception \& Psychophysics, 1972, 11, 139-142.

Dodd, D. H., \& Schultz, R. F., Jr. Computational procedure for estimating magnitude of effect for some analysis of variance designs. Psychological Bulletin, 1973, 79, 391-393.

Filbey, R. A., \& Gazzaniga, M.S. Splitting the normal brain with reaction time. Psychonomic Science, 1969, 17, 335-336.

Kendler, H. H., \& Kendler, T. S. Vertical and Horizontal processes in problem solving. Psychological R eview, 1962, 69, 1-16.

Kendler, H. H., Kendler, T. S., \& Saunders, J. Reversal and partial reversal shifts with verbal material. Journal of Verbal Learning \& Verbal Behavior, 1967, 6, 117-127. 
Kendler, H. H., Kendler, T. S., \& Wells, D. Reversal and nonreversal shifts in nursery school children. Journal of Comparative \& Physiological Psychology, 1960, 53, 83-87.

Kendler, T. S. Verbalization and optional reversal shifts among kindergarten children. Journal of Verbal Learning \& Verbal Behavior, 1964, 3, 428-433.

McAdam, D. W., \& Whitaker, H. A. Language production:
Electroencephalographic localization in the normal human brain. Science, $1971,172,499-502$.

Penfield, W., \& Roberts, L. Speech and brain mechanisms. Princeton: Princeton University Press, 1959.

(Received for publication August 21, 1974.)

Bulletin of the Psychonomic Society

1975, Vol. 5 (1), 15-17

\title{
Going beyond tests of significance: Is psychology ready?*
}

\author{
D. JAMES DOOLING and JOSEPH H. DANKS \\ Kent State University, Kent, Ohio 44242
}

\begin{abstract}
A criticism is offered for the recent efforts to encourage psychologists to report the proportion of variance accounted for $\left(\omega^{2}\right)$ by analysis of variance effects. Few psychological experiments employ designs that allow legitimate inferences as to the strength of particular effects. As such, $\omega^{2}$ is a descriptive statistic that is extremely limited in its usefulness. It is suggested that a widespread reporting of $\omega^{2}$ in psychology is not only unnecessary, but could also be misleading.
\end{abstract}

In the past decade, some psychologists have criticized the mere reporting of significance levels in analysis-of-variance designs. According to this view, it is also important, even essential, to report the "strength" of the significant effects that have been obtained. In addition to the reporting of F-ratios, psychologists have been urged to compute a statistic that reflects the proportion of variance accounted for by a given effect $\left(\omega^{2}\right)$. A source most influential in this regard was Hays's Statistics for psychologists (1963). His argument can be summarized as follows: "The occurrence of a significant result says nothing at all about the strength of the association between treatment and score. A significant result leads to the inference that some association exists, but in no sense does this mean that an important degree of association necessarily exists. Conversely, evidence of a strong statistical association can occur in data even when the results are not significant [1963, p. 342]." More recently, Vaughan and Corballis (1969) called

*We are grateful to Clyde Hendrick, Roy Lachman, Roy S. Lilly, and Terry J. Spencer for their comments on an earlier version of this manuscript. attention to the fact that research psychologists have not heeded Hays's advice. They contended that lack of such information in published articles is a serious problem and that "a change of attitude is required [1969, p. 204]." Following Vaughan and Corballis four years later, Dodd and Schultz (1973) echo their cry, providing computational procedures in an attempt to "encourage researchers to include estimates of magnitude of effects [p. 395]."

We take a position in opposition to the opinions expressed above, though we find merit in the positions stated. Our comment does not fault either the reasoning of Hays or the computational formulas provided by either Vaughan and Corballis or by Dodd and Schultz. We find the logic of the Hays comment impeccable and agree that knowledge of the strength of association would add new information not given by the usual report of significance levels alone. Our quarrel is rather with a possible (and we think, probable) misinterpretation of the proportion of variance accounted for measure $\left(\omega^{2}\right)$ when reported in journals and read by psychologists. In addition, we argue that the 